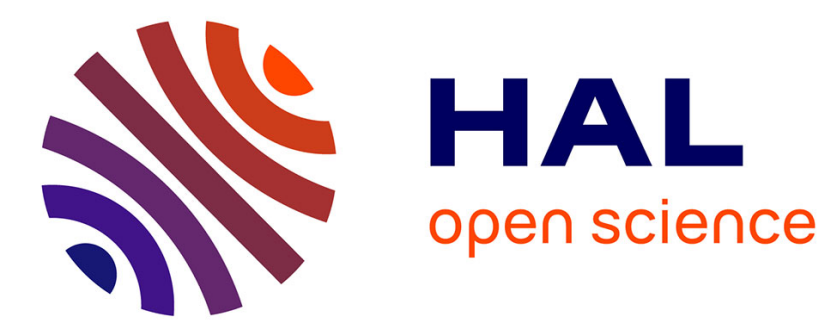

\title{
Dispositif digital d'étalonnage d'un champ magnétique application à la spectroscopie R.P.E.
}

\author{
P. Ackermann, J.P. Imbaud, J.P. Plumey, G. Rives
}

\section{To cite this version:}

P. Ackermann, J.P. Imbaud, J.P. Plumey, G. Rives. Dispositif digital d'étalonnage d'un champ magnétique application à la spectroscopie R.P.E.. Revue de Physique Appliquée, 1976, 11 (5), pp.629-637. 10.1051/rphysap:01976001105062900 . jpa-00244098

\section{HAL Id: jpa-00244098 https://hal.science/jpa-00244098}

Submitted on 1 Jan 1976

HAL is a multi-disciplinary open access archive for the deposit and dissemination of scientific research documents, whether they are published or not. The documents may come from teaching and research institutions in France or abroad, or from public or private research centers.
L'archive ouverte pluridisciplinaire HAL, est destinée au dépôt et à la diffusion de documents scientifiques de niveau recherche, publiés ou non, émanant des établissements d'enseignement et de recherche français ou étrangers, des laboratoires publics ou privés. 


\author{
Classification \\ Physics Abstracts \\ 0.667
}

\title{
DISPOSITIF DIGITAL D'ÉTALONNAGE D'UN CHAMP MAGNÉTIQUE APPLICATION A LA SPECTROSCOPIE R.P.E.
}

\author{
P. ACKERMANN, J. P. IMBAUd, J. P. PLUMEY et G. RIVES \\ Laboratoire d'Electronique et Résonance Magnétique (*) \\ Université de Clermont, B. P. 45, 63170 Aubière, France
}

(Reçu le 13 janvier 1976, accepté le 4 mai 1976)

\begin{abstract}
Résumé. - L'appareillage présenté assure le marquage automatique du champ magnétique d'un spectrographe R. P. E. fonctionnant en bande X. Il permet d'obtenir directement un repérage du champ magnétique en marge de l'enregistrement graphique d'un spectre de R.P. E., ou de tout autre phénomène dépendant du champ magnétique. Ce marquage est exécuté tous les gauss, avec un repérage des dizaines de gauss ; sa précision est de l'ordre de $1 / 100$ gauss et il fonctionne jusqu'à des vitesses de variation du champ magnétique de 25 gauss par minute.
\end{abstract}

\begin{abstract}
A device is described which enables the automatic calibration and tracking of the magnetic field of an E. P. R. spectrometer. The device allows one to plot a scale of the magnetic field, in the margin of the chart paper along with the investigated E. P. R. spectrum-or any phenomena depending on the magnetic field. The scale is equal to 1 gauss, with a special mark every ten gauss. The instrument operates with a maximum sweep rate of 25 gauss/minute, and the accuracy is approximatively $1 / 100$ gauss.
\end{abstract}

1. Introduction. - La fonction de l'appareillage décrit est le repérage du champ magnétique continu d'un électroaimant; il a été réalisé pour s'intégrer dans un dispositif de résonance paramagnétique électronique (R. P. E.), afin de mesurer avec précision les paramètres de couplages hyperfins des radicaux semiquinones et nitroxydes.

Le principe de l'appareillage est l'asservissement sur le champ magnétique $B_{0}$ d'une fréquence $v$ au moyen de la résonance magnétique nucléaire (R.M.N.) [1], [2]. $\mathrm{Au}$ centre de la raie de résonance la fréquence $F_{0} \mathrm{du}$ champ d'irradiation est liée à la valeur du champ magnétique directeur $B_{0}$ par la relation:

$$
h F_{0}=g_{\mathrm{N}} \beta_{\mathrm{N}} B_{0}
$$

où $g_{\mathrm{N}}$ est le facteur de décomposition spectrale et $\beta_{\mathrm{N}}$ le magnéton nucléaire. Le dispositif réalisé asservit la fréquence $F_{0}$ de telle façon que la condition de résonance soit réalisée en permanence pendant la variation linéaire du champ $B_{0}$.

Le champ radiofréquence est obtenu à partir d'un oscillateur autodyne dans lequel le passage par la résonance provoque une variation du coefficient de surtension du circuit oscillant et, par conséquent, une variation du niveau des oscillations radiofréquence. Un traitement de ce signal par détection synchrone permet

(*) Equipe de Recherche Associée au C. N. R. S. n 90.

ReVUe de PHYSIQUe APPLIQUÉe. - T. 11, No 5, SEPTEMBRe 1976. d'obtenir un signal en tension représentant la dérivée première de la courbe d'absorption $\chi^{\prime \prime}$. Lorsque le spectrographe fonctionne en champ variable à fréquence fixe, le signal de détection représenté sur la figure 1 peut être assimilé à la réponse d'un discriminateur.

Nous pouvons considérer que dans toute la partie AB de la courbe, un écart $\Delta B_{0}$ du champ magnétique directeur provoque à la sortie du détecteur synchrone une variation de tension $\Delta e$ de module proportionnel à $\Delta B_{0}$. De plus, le signe de $\Delta e$ indique le sens dans lequel le champ a varié ; nous obtenons ainsi un signal d'erreur qui donne une image quantitative de l'éloignement par rapport à la condition de résonance. Lorsque le champ varie, le signal d'erreur qui apparaît fait varier la fréquence de l'oscillateur au moyen d'une diode à capacité variable.

La fréquence de l'oscillateur est ainsi une image fidèle du champ magnétique directeur grâce au système d'asservissement. Elle est mesurée par un ensemble de compteurs dont le temps de comptage est fixé par une horloge à quartz. Des circuits logiques permettent d'obtenir un signal dès que la fréquence a varié de $4257,7 \mathrm{~Hz}$, ce qui correspond à une variation du champ magnétique de 1 gauss.

2. Description des circuits du système asservi. - La figure 2 représente le schéma synoptique du dispositif 

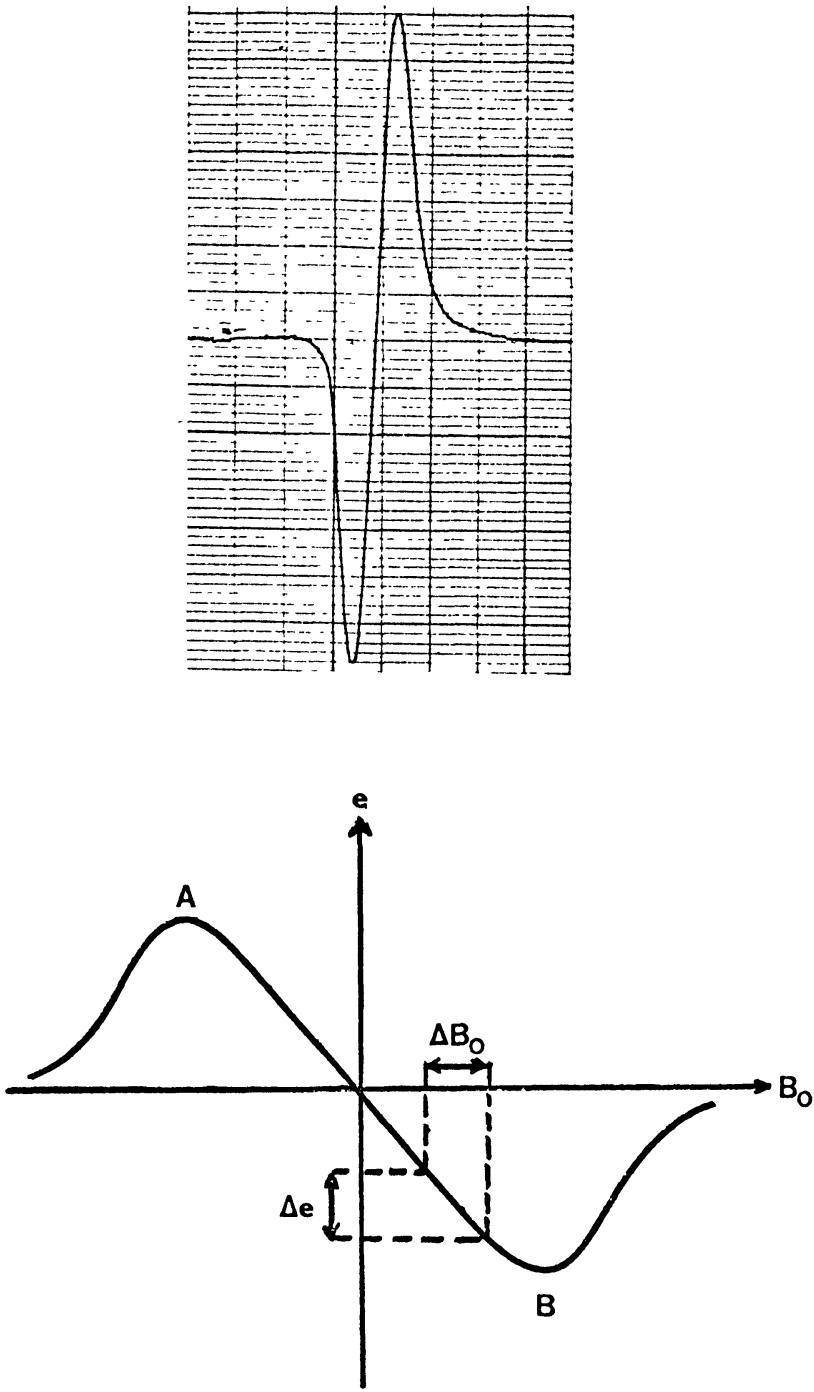

Fig. 1. - Signal R. M. N. et courbe de discrimination du détecteur synchrone.

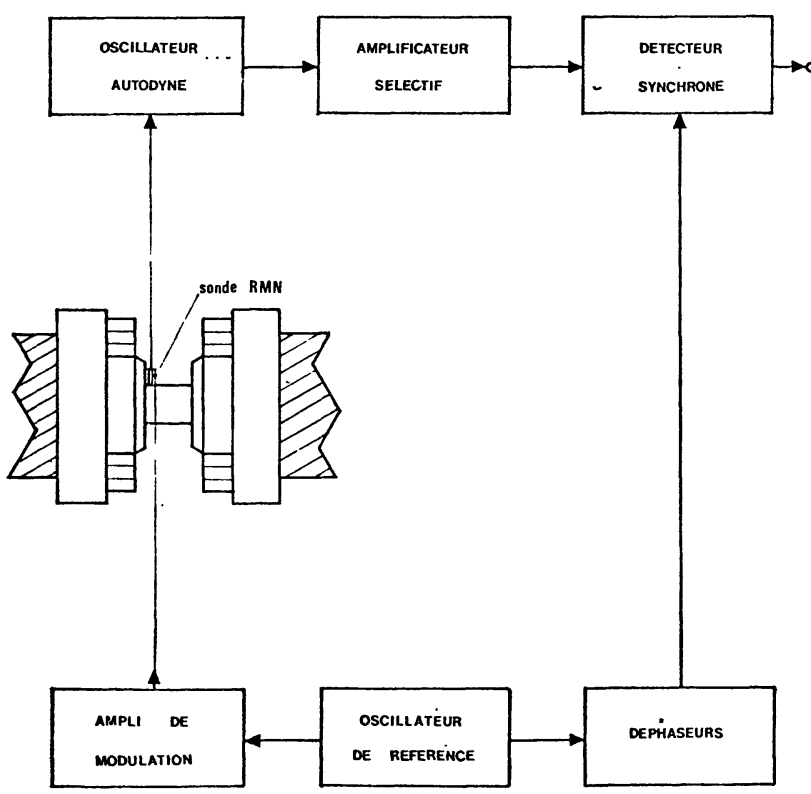

FIG. 2. - Spectrographe R. M. N.
R. M. N. Le champ magnétique du spectrographe R. P. E. variant au voisinage de 3400 gauss, la condition de résonance fixe la fréquence de l'oscillateur autour de 14,3 $\mathrm{MHz}$. Une excursion en fréquence de l'ordre de un mégahertz couvre largement le domaine d'exploration du champ directeur.

2.1 Sonde R. M. N. - La partie centrale de l'entrefer de l'électroaimant est occupée par la cavité hyperfréquence du spectrographe R. P. E. La sonde R. M. N. est donc placée dans un champ un peu différent et plus inhomogène. Pour ne pas trop élargir la raie de résonance l'échantillon choisi (eau dopée en sulfate de cuivre) est de faibles dimensions $\left(0,1 \mathrm{~cm}^{3}\right)$.

2.2 Oscillateur [3], [4], [5], [6]. - Il s'agit de l'oscillateur Robinson (Fig. 3) qui présente à la fois la sensibilité d'un oscillateur séparé (Rollin) et l'immunité au bruit d'un oscillateur marginal (Pound \& Knight). Il se compose d'un étage amplificateur à grand gain, large bande passante $(1 \mathrm{MHz})$ et faible bruit $(\mathrm{E} 810 \mathrm{~F})$ et d'un étage limiteur (6 AK 5). Le circuit oscillant est constitué de la self-inductance de la sonde accordée avec une capacité fixe et la diode à capacité variable. Le passage par la résonance se traduit par une variation du facteur de surtension $Q$ du circuit oscillant : $\Delta Q=-4 \pi \eta \chi^{\prime \prime} Q^{2}$ où $\eta$ est le facteur de remplissage.

La variation d'amplitude du signal radiofréquence est alors proportionnelle à l'absorption $\chi^{\prime \prime}$; elle est détectée par le tube limiteur dont la grille et la cathode se comportent comme une diode de détection. Une cellule R. C. permet d'éliminer la composante radiofréquence.

Notons que sous l'aspect du rapport signal/bruit, il serait probablement plus avantageux d'utiliser un oscillateur à F. E. T., par exemple celui de Colligiani [7] [8], au lieu de l'oscillateur Robinson.

2.3 Détection synchrone. - La détection synchrone qui suit l'oscillateur, permet d'améliorer notablement le rapport signal/bruit. Elle permet en outre d'obtenir directement la dérivée première de $\chi^{\prime \prime}$, indispensable au fonctionnement du dispositif : le champ directeur $B_{0}$ est modulé par un champ basse fréquence, petit devant $B_{0}$

$$
b=b_{\mathrm{m}} \sin \omega t .
$$

Un amplificateur sélectif à la pulsation $\omega$ fournit un signal de sortie proportionnel à :

$$
\left(\frac{\mathrm{d} \chi^{\prime \prime}}{\mathrm{d} B}\right) \sin (\omega t+\varphi)
$$

où $\varphi$ est le déphasage de ce signal par rapport au signal de référence également utilisé pour moduler le champ magnétique.

Le circuit de détection synchrone effectue le produit de ces deux signaux et fournit, grâce à un filtre passebas, un signal proportionnel à :

$$
\left(\frac{d \chi^{\prime \prime}}{d B}\right) \cos \varphi
$$




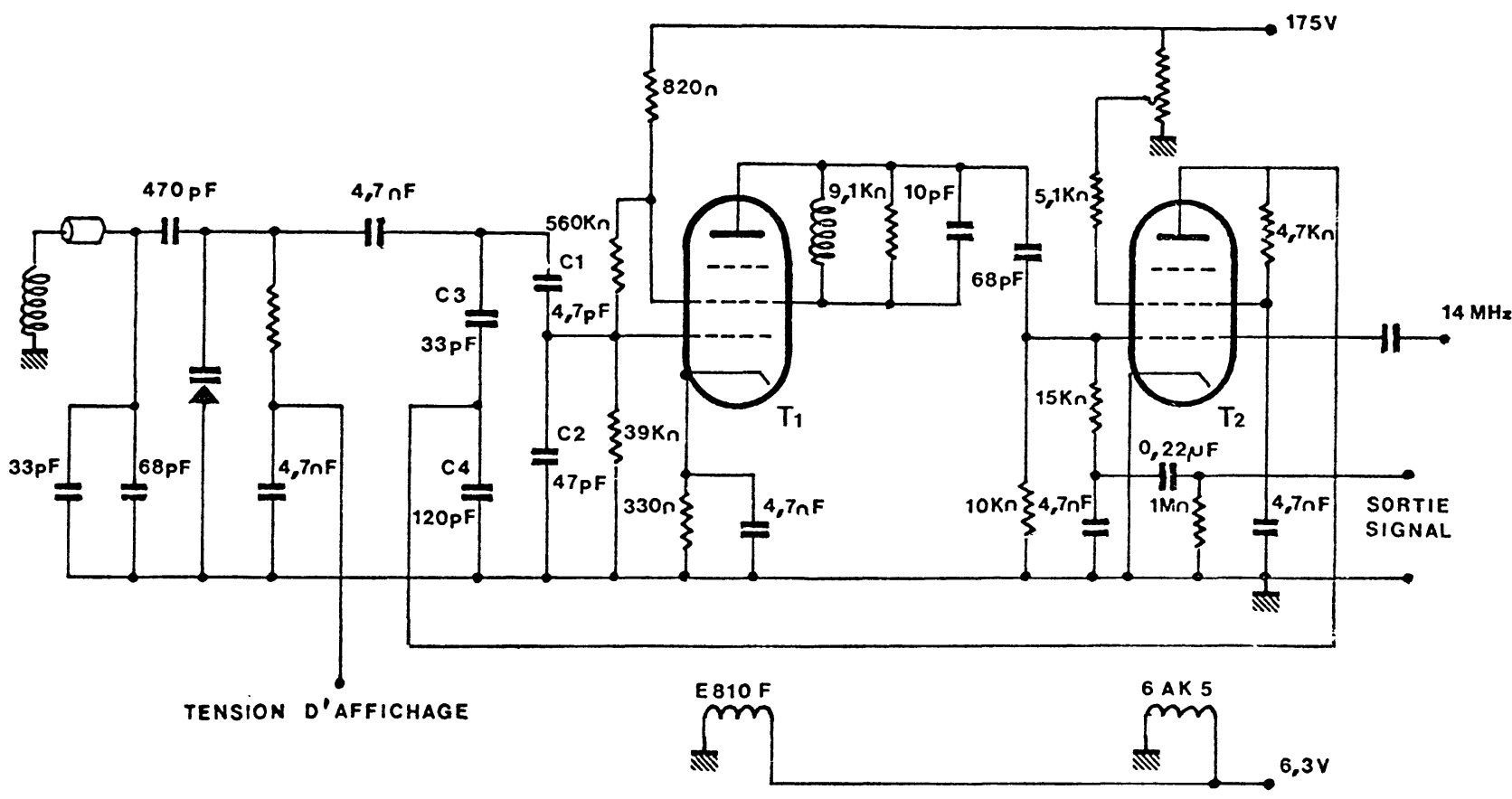

Fig. 3. - Oscillateur Robinson.

La fréquence de l'oscillateur de référence $\omega / 2 \pi$ doit être suffisamment faible pour tenir compte des protons de l'échantillon, mais aussi suffisamment grande pour permettre une largeur de bande compatible avec une réponse assez rapide du système. De plus il est préférable d'éviter la présence d'harmoniques du secteur $50 \mathrm{~Hz}$ dans la bande passante. Ces différentes contraintes ont conduit à fixer la fréquence de l'oscillateur de référence à $380 \mathrm{~Hz}$. L'amplitude du champ de modulation $b_{\mathrm{m}}$ doit être petite devant la largeur de raie expérimentale (maximum 500 mgauss) ; la valeur utilisée est de quelques dizaines de milligauss).

3. Représentation du système asservi. - La nature même du dispositif, dont la conception repose sur le phénomène de résonance magnétique nucléaire, rend difficile sa représentation sous la forme habituelle du schéma fonctionnel de l'asservissement.

Le principe de fonctionnement de l'appareillage est d'asservir la fréquence $F$ de l'oscillateur à la valeur du champ magnétique $B_{0}$, ce qui devrait conduire à prendre $B_{0}$ comme grandeur d'entrée et $F$ comme grandeur de sortie.

Ces deux grandeurs étant de natures différentes nous prenons pour grandeur d'entrée la fréquence $F_{0}$ liée à $B_{0}$ par la condition de résonance magnétique nucléaire, le système de spins nucléaires fonctionnant comme un convertisseur champ-fréquence.

La chaîne directe de l'asservissement (Fig. 4) est composée de la détection synchrone, de la diode à capacité variable et l'oscillateur Robinson. Le détecteur synchrone se comporte comme un discriminateur qui fournit une tension e dont les variations $\Delta e$ sont images des variations $\Delta B_{0}$ ou encore image de l'écart en fréquence

$$
\Delta F=F_{0}-F .
$$

Cette différence de fréquence a en fait un caractère purement formel ; elle n'est pas réalisée physiquement en un endroit précis de la chaîne.

Au signal de sortie du détecteur synchrome, se superpose la tension d'affichage de la diode à capacité variable dont la valeur $V_{0}$ correspond à la fréquence centrale $F_{0}$. La somme de ces deux tensions commande l'accord du circuit oscillant de l'oscillateur autodyne et détermine ainsi la fréquence $F$.

L'étude physique de l'asservissement représenté par un tel schéma fonctionnel est difficile parce que les grandeurs d'entrée et de sortie sont des fréquences; mais le problème peut être résolu en remarquant que la tension d'affichage $V_{0}$ apparaît comme une entrée secondaire. L'asservissement peut donc être représenté par le schéma fonctionnel de la figure 5 ; le signal de sortie est alors celui qui est fourni par le détecteur synchrone. C'est cette représentation que nous considérons pour l'étude expérimentale du système.

4. Identification du système $\left({ }^{1}\right)$. - La fonction de transfert en boucle ouverte a été déterminée par analyse harmonique à l'aide d'un analyseur de fonction de transfert. Pour cela, une tension sinusoïdale est mise en série avec la tension d'affichage de la diode à capacité variable, réglée pour obtenir la condition de résonance

(1) Les notations et le formalisme sont ceux utilisés usuellement en théorie des systèmes asservis (cf. par exemple [9], [10], [11]). 


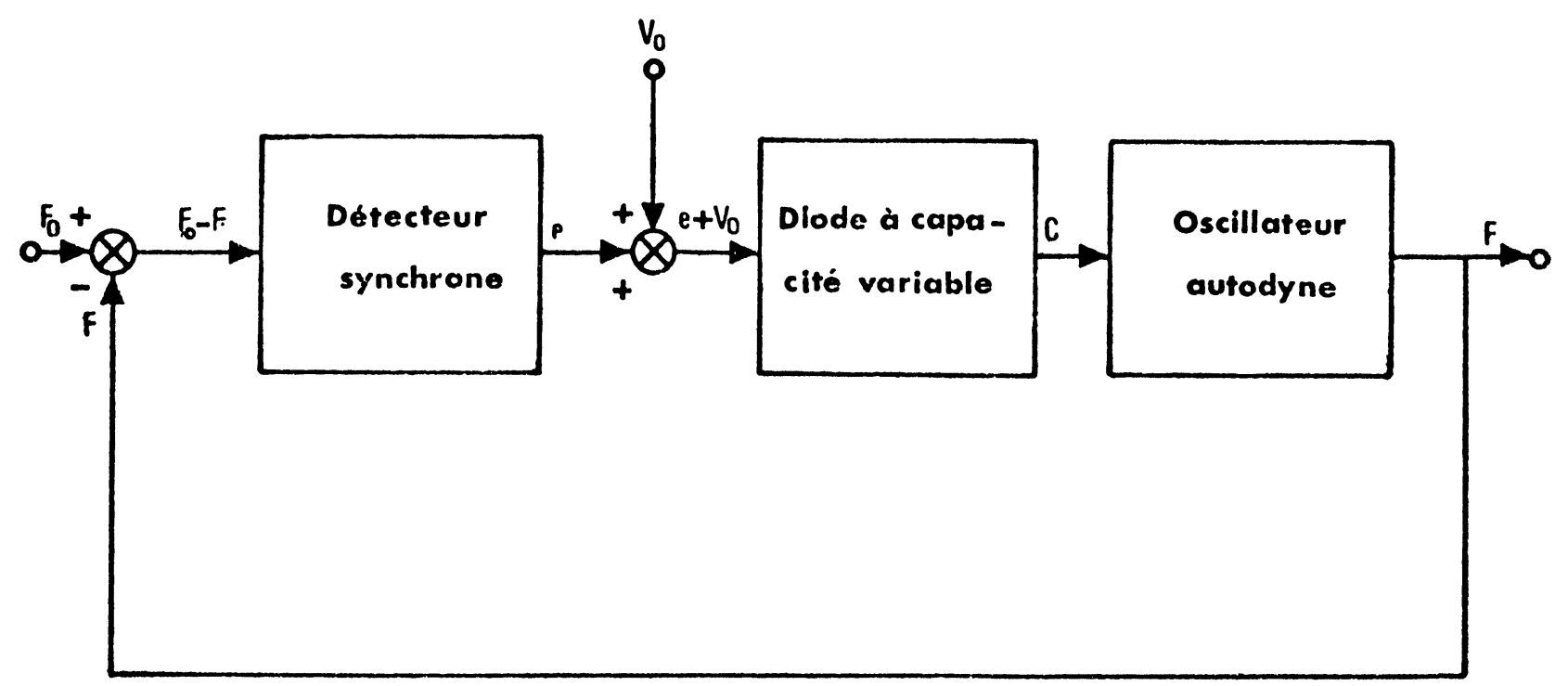

FIG. 4. - Représentation de l'asservissement.

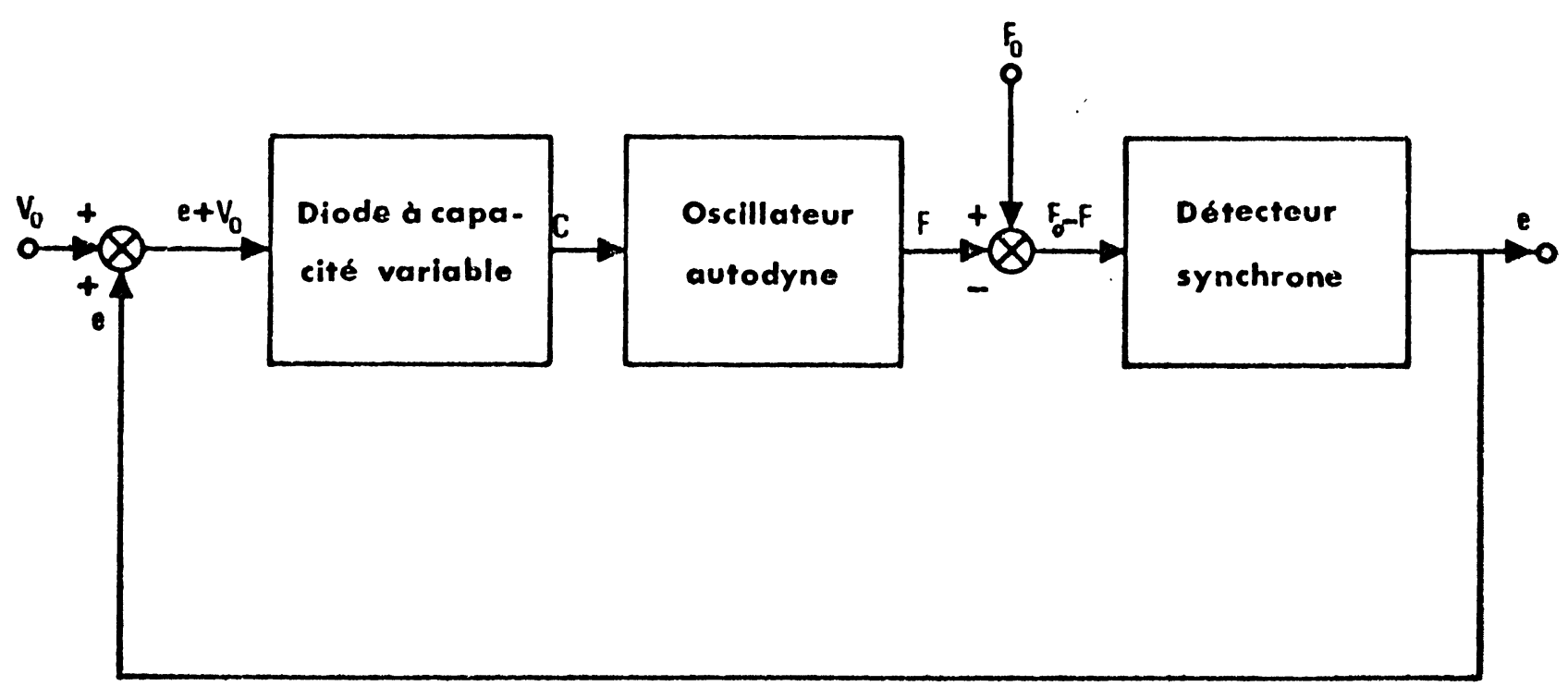

FIG. 5. - Etude par rapport à l'entrée secondaire.

nucléaire au centre de la raie. L'amplitude du signal appliqué est faible, afin de rester constamment dans la partie linéaire de la courbe de discrimination du détecteur synchrone (Fig. 1). Le signal de sortie du système ainsi analysé est celui du détecteur synchrone. On note que cette étude est statique puisqu'elle nécessite une valeur fixe du champ magnétique directeur $B_{0}$. Le système présente l'allure d'un système du troisième ordre ce qui correspond à une fonction de transfert en boucle ouverte de la forme

$$
T(p)=\frac{K}{\left(1+\tau_{1} p\right)\left(1+\tau_{2} p\right)\left(1+\tau_{3} p\right)}
$$

où $K$ est le gain statique et $\tau_{1}, \tau_{2}, \tau_{3}$ sont les constantes de temps du système. Leurs valeurs, trouvées pour notre dispositif à partir de la courbe expérimentale d'amplitude tracée dans le plan de Bode, sont :

$$
\tau_{1}=0,106 \mathrm{~s}, \tau_{2}=0,059 \mathrm{~s}, \tau_{3}=0,044 \mathrm{~s} .
$$

Le gain $K$ est pris suffisamment petit pour avoir la possibilité de corriger le système et pour éviter le phénomène de pompage.

$$
K \mathrm{db}=11 \mathrm{db} .
$$

Notons que la valeur $K$ est essentiellement liée aux conditions expérimentales, en particulier au signal de résonance nucléaire ; ceci n'est pas un inconvénient puisque une variation de $K$ se traduit simplement par une translation du lieu de la fonction de transfert dans le plan de Black, à condition de rester dans le domaine de linéarité de la courbe de discrimination.

Le système étant à retour unitaire, les valeurs du facteur de résonance $Q$ et de la fréquence $F_{\mathrm{r}}$ du système en boucle fermée sont lues directement sur l'abaque de Black (Fig. 6) :

$$
Q=12 \mathrm{db}, \quad F_{\mathrm{r}}=3,5 \mathrm{~Hz} .
$$




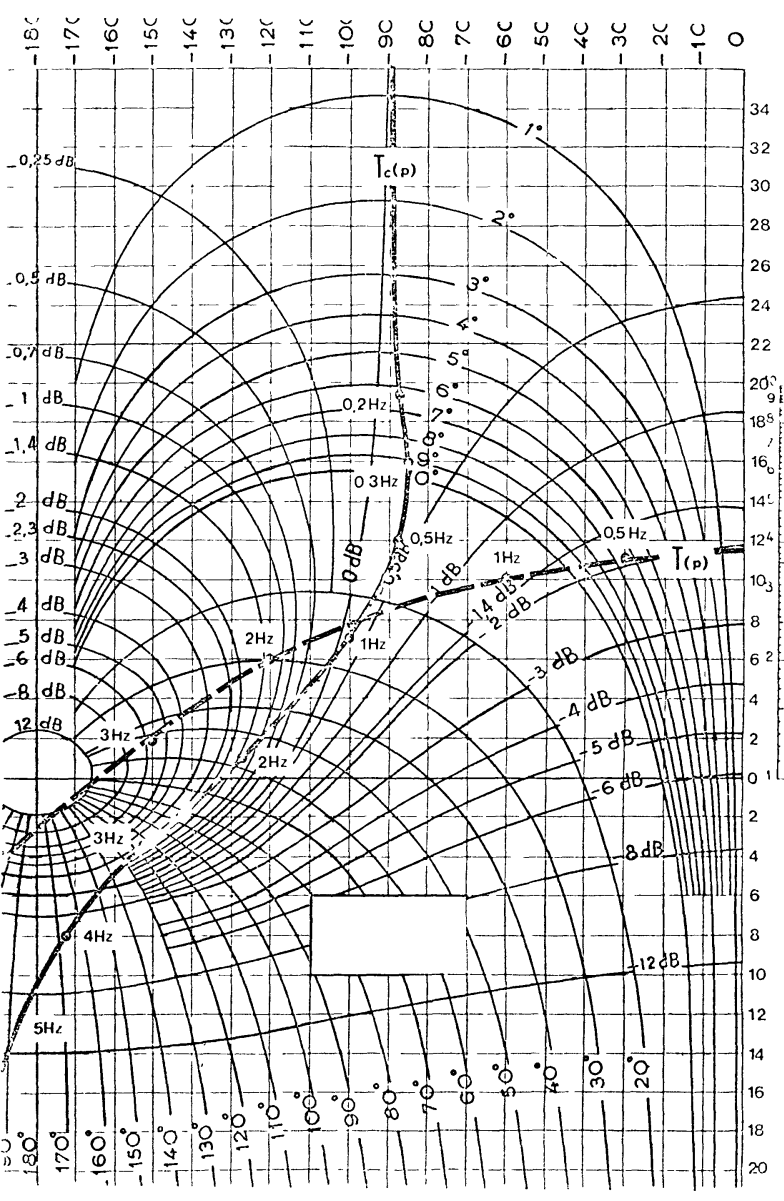

FiG. 6. - Lieu de Black du système non corrigé : $T(p)$. Lieu de Black du système corrigé par avance de phase et action proportionnelle et intégrale : $T_{\mathrm{c}}(p)$.

5. Correction du système asservi. - La recherche d'un compromis entre la stabilité et la précision dynamique conduit à prendre un facteur de résonance de valeur égale à $2,3 \mathrm{db}$. Pour arriver à ce résultat, il a donc fallu augmenter la phase au voisinage de la résonance grâce à un correcteur à avance de phase (Fig. 7) de fonction de transfert $D_{1}(p)$

$$
D_{1}(p)=\frac{1}{a} \frac{1+a \tau_{4} p}{1+\tau_{4} p}
$$

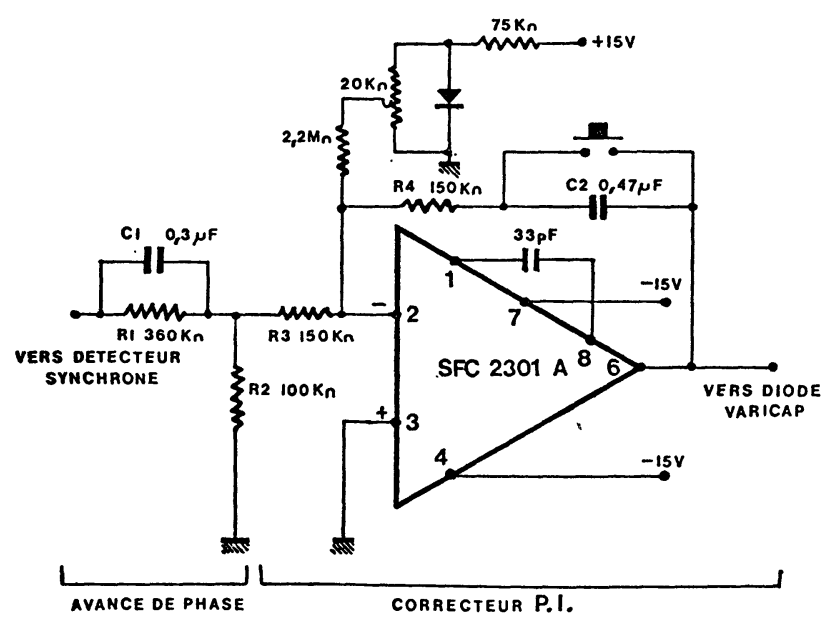

Fig. 7. - Correcteur à avance de phase et correcteur P.I. avec

$$
\tau_{4}=\frac{R_{1} R_{2}}{R_{1}+R_{2}} C_{1}, \quad a=1+\frac{R_{1}}{R_{2}} .
$$

L'avance de phase maximum est donnée par :

$$
\Delta \varphi_{\mathrm{M}}=\operatorname{Arcsin} \frac{a-1}{a+1} .
$$

Pour une fréquence $F_{1}$ :

$$
F_{1}=\frac{1}{2 \pi \tau_{1} \sqrt{a}} .
$$

Le choix des valeurs de $R_{1}$ et $R_{2}$ permet d'obtenir l'avance de phase désirée et celui de $C$ permet de fixer $F_{1}$ au voisinage de $F_{r}$. Pour :

$$
R_{1}=360 \mathrm{~K} \Omega, \quad R_{2}=100 \mathrm{~K} \Omega \text { et } C_{1}=0,3 \mu \mathrm{F} .
$$

On obtient :

$$
\Delta \varphi_{\mathrm{M}}=40^{\circ} \text { à la fréquence } F_{1}=3,2 \mathrm{~Hz} .
$$

Le système ainsi corrigé présente une bonne stabilité et une rapidité satisfaisante; par contre la plage d'asservissement est limitée comme le montre l'enregistrement de la figure 8 . Ceci provient du fait que le champ magnétique directeur $B_{0}$ est balayé suivant une fonction rampe. La fréquence $F_{0}$ correspondant au centre de la raie de résonance est liée linéairement à $B_{0}$ par la condition de résonance :

$$
h F_{0}=g_{\mathrm{N}} \beta_{\mathrm{N}} B_{0} \text {. }
$$

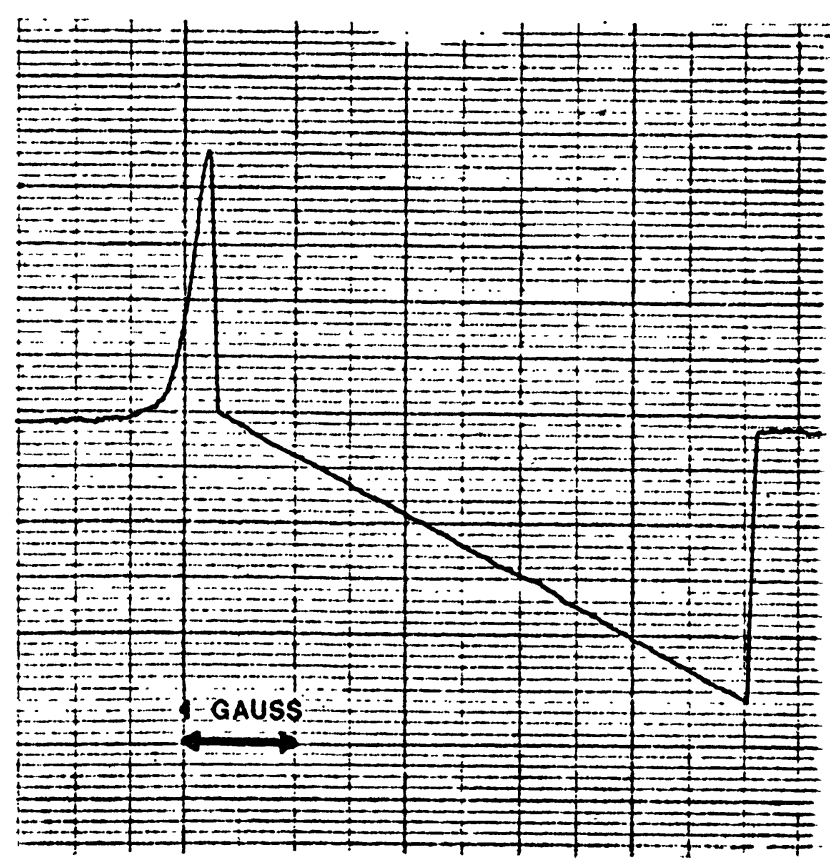

Fig. 8. - Réponse à une rampe sans correcteur P. I

La fréquence $F_{0}$ varie donc aussi selon une fonction: rampe que l'on peut représenter par la figure 9 et. exprimer sous la forme analytique :

$$
F_{0}=\left(F_{0}^{0}+\alpha t\right) \cdot u(t)
$$




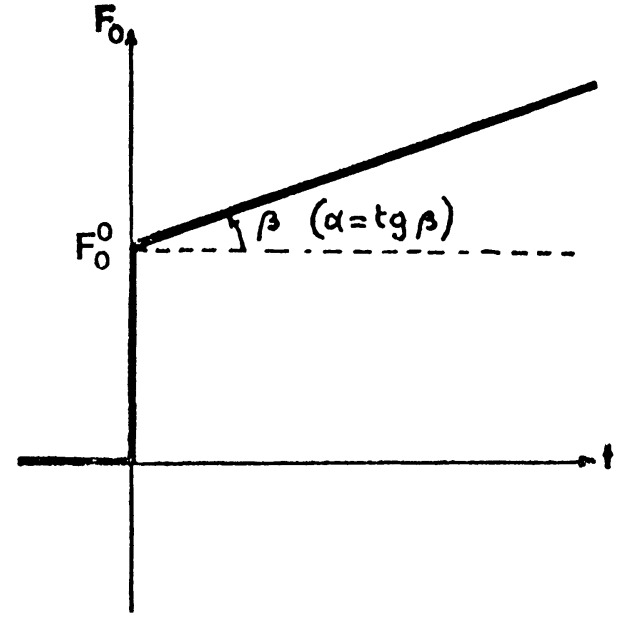

Fig. 9. - Représentation de la variation de la fréquence de résonance nucléaire $F_{0}$ pendant le balayage du champ magnétique.

de transformée de Laplace :

$$
F_{0}(p)=\frac{F_{0}^{0}}{p}+\frac{\alpha}{p^{2}}
$$

$u(t)$ est la fonction d'Heaviside forme analytique de l'échelon unité.

En se reportant au schéma fonctionnel de la figure 4 la transformée de Laplace du signal d'erreur s'écrit d'après (3.1)

$$
\varepsilon(p)=F_{0}(p)-F(p)
$$

avec

$$
F(p)=G_{2}(p) V_{0}+G_{1}(p) G_{2}(p) \varepsilon(p)
$$

où $G_{1}(p)$ est la fonction de transfert du détecteur synchrone et $G_{2}(p)$ celle de l'ensemble constitué par la diode à capacité variable et l'oscillateur autodyne.

En rapprochant (5.6) et (5.7)

$$
\varepsilon(p)=\frac{F_{0}(p)}{1+G_{1}(p) G_{2}(p)}-\frac{V_{0} G_{2}(p)}{1+G_{1}(p) G_{2}(p)}
$$

ou encore en remarquant au $G_{1}(p) G_{2}(p)=T(p)$

$$
\varepsilon(p)=\frac{F_{0}(p)}{1+T(p)}-\frac{V_{0}}{1+T(p)} G_{2}(p) .
$$

En reportant la valeur de $F_{0}(p)$ donnée par (5.5) on obtient :

$$
\begin{aligned}
\varepsilon(p)=\frac{F_{0}^{0}}{p[1+T(p)]}+ & \frac{\alpha}{p^{2}[1+T(p)]}- \\
& -\frac{V_{0}}{1+T(p)} \cdot \frac{T(p)}{G_{1}(p)} .
\end{aligned}
$$

D'après le théorème de la valeur finale :

$$
\lim _{t \rightarrow \infty} \varepsilon(t)=\lim _{p \rightarrow \infty} p \varepsilon(p)
$$

$$
\begin{array}{r}
\lim _{t \rightarrow \infty} \varepsilon(t)=\lim _{p \rightarrow \infty} \frac{F_{0}^{0}}{1+T(p)}+\frac{\alpha}{p[1+T(p)]}- \\
-\frac{p V_{0}}{1+T(p)} \cdot \frac{T(p)}{G_{1}(p)} .
\end{array}
$$

La fonction de transfert $T(p)$ n'a pas de pôle à l'origine, le $2^{\mathrm{e}}$ terme de l'expression (5.11) diverge; l'erreur stationnaire correspondant au balayage du champ directeur croît jusqu'à ce que le système décroche. Un pôle à l'origine est introduit grâce à un correcteur à action proportionnelle et intégrale P. I. (Fig. 7), de fonction de transfert :

$$
D_{2}(p)=\frac{1+a \tau_{5} p}{\tau_{5} p}
$$

avec :

$$
a=\frac{R_{3}}{R_{4}} \quad \text { et } \quad \tau_{5}=R_{4} C_{2} .
$$

En prenant $R_{3}=R_{4}$ :

$$
a=1 \quad \text { et } \quad D_{2}(p)=\frac{1+\tau_{5} p}{\tau_{5} p} .
$$

Ce correcteur est placé à la sortie de la détection synchrone ; il suffit donc de remplacer $G_{1}(p)$ par

$$
G_{1}(p) \cdot \frac{1+\tau_{5} p}{\tau_{5} p}
$$

dans l'expression (5.11) qui donne :

$$
\lim _{t \rightarrow \infty} \varepsilon(t)=\frac{\alpha \tau_{5}}{K} .
$$

Pour tenir compte de l'influence du correcteur à avance de phase il faut substituer dans (5.11) $G_{1}(p) D_{1}(p) D_{2}(p)$ à $G_{1}(p)$ ce qui conduit à :

$$
\lim _{t \rightarrow \infty} \varepsilon(t)=\frac{\alpha a \tau_{5}}{K}
$$

qui est l'erreur stationnaire du second ordre du système corrigé ou erreur de trainage.

Remarquons que cette erreur n'est pas nulle mais qu'elle est cependant petite et surtout constante ; elle n'intervient donc pas dans la précision du système puisque seules les variations du champ directeur $B_{0}$ interviennent dans la mesure des constantes de couplage.

Dans la réalisation pratique, un courant très faible est injecté dans l'amplificateur opérationnel pour éviter que celui-ci ne dérive en intégrant le courant de décalage. De plus un bouton pressoir permet de courtcircuiter le condensateur $\left(\mathrm{C}_{2}\right)$ avant de boucler l'asservissement.

Pour $R_{3}=R_{4}=140 \mathrm{k} \Omega$ et $C=0,47 \mu \mathrm{F}$, on obtient :

$$
\tau_{5}=70,5 \times 10^{-3} \mathrm{~s} .
$$

Notons que ce correcteur inverse la phase ; il y a lieu d'en tenir compte dans le bouclage du système, ainsi d'ailleurs que du réglage de la phase du détecteur synchrone.

En définitive la fonction de transfert du système corrigé par réseau à avance de phase et correcteur P. I. peut s'écrire :

$$
T_{\mathrm{c}}(p)=T(p) D_{1}(p) D_{2}(p) .
$$


Sa représentation sur l'abaque de Black (Fig. 6) permet de déduire directement la valeur du facteur de résonance $Q$ et de la fréquence de résonance $F_{\mathrm{r}} \mathrm{du}$ système en boucle fermée à retour unitaire

$$
Q=2,3 \mathrm{db}, \quad F_{\mathrm{r}}=3 \mathrm{~Hz} .
$$

La plage d'asservissement du système ainsi corrigé n'est limitée que par l'excursion en fréquence de l'oscillateur Robinson $(0,7 \mathrm{MHz})$; la variation correspondante du champ magnétique est de 165 gauss.

6. Système de marquage. - Le but n'étant pas exactement de connaître la fréquence de l'oscillateur autodyne mais bien plutôt la valeur du champ magnétique dont elle est l'image, la fréquence de l'horloge a été fixée à $42577 \mathrm{~Hz}$ ce qui permet d'obtenir un temps de comptage de (1/42 577) s.

A une fréquence de $4257,7 \mathrm{~Hz}$ correspond par la condition de résonance $\left(h F_{0}=g_{\mathrm{N}} \beta_{\mathrm{N}} B_{0}\right)$ un champ magnétique de 1 gauss. Le nombre de périodes mesuré correspondant à une telle fréquence est donc :

$$
\frac{4257,7}{42,577}=100 \text {. }
$$

Le résultat du comptage n'est pas la valeur de la fréquence mais celle du champ magnétique qui lui correspond, c'est-à-dire 1,00 gauss.

Le choix du temps de comptage égal à la durée de 1000 impulsions d'horloge a été guidé par la recherche d'un compromis entre la précision de la mesure de fréquence et la vitesse de cette fréquence, liée directement au balayage du champ magnétique. Un champ de 1 gauss correspond à 100 impulsions, la valeur du champ magnétique est donc connue à $1 / 100$ gauss près. D'autre part la période de comptage comprend le temps de comptage (1 000 impulsions d'horloge) et le temps nécessaire à la lecture du résultat et la remise à zéro de l'ensemble des circuits de comptage. Le temps doit être le plus court possible puisqu'il faut que pendant la durée totale de la période de comptage le champ magnétique ait varié de moins de $1 / 100$ gauss ; il a été fixé à une seule période d'horloge. Le champ magnétique est ainsi mesuré au centième de gauss près pour une vitesse de balayage inférieure à 25 gauss/minute.

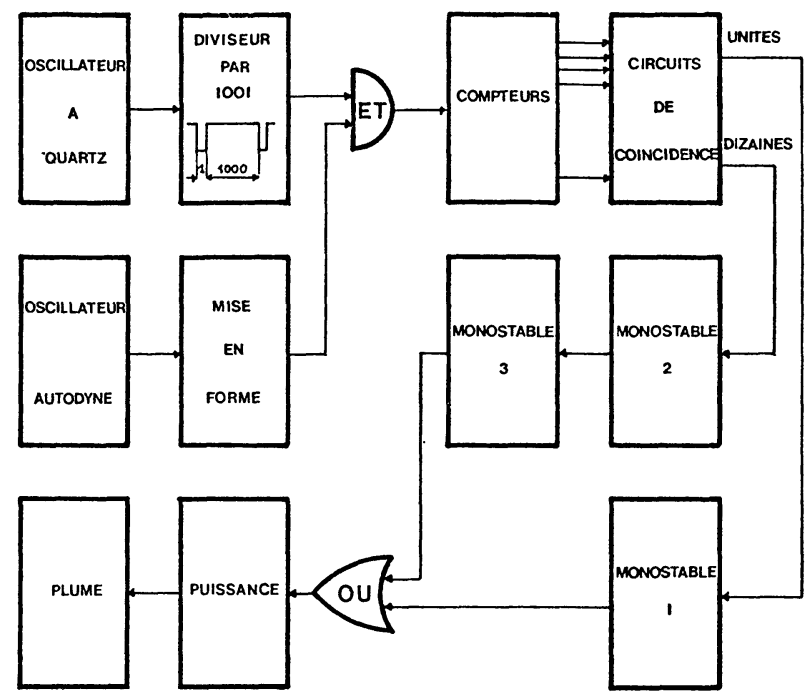

Fig. 10. - Schéma synoptique du marqueur.

La figure 10 donne le schéma fonctionnel du système de marquage. La fréquence du signal $\mathrm{H}$. F. (14 MHz) est mesurée à l'aide d'une série de compteurs (circuits 5 , 6 et 7 de la figure 11) pendant le temps d'ouverture d'une porte ET commandée à partir d'une horloge à quartz par un diviseur par 1001. Ce diviseur est réalisé à l'aide de quatre diviseurs par 10 (circuits $1,2,3$ et 4 de la figure 11). La sortie $A$ du circuit 4 est à l'état bas pendant 1000 périodes d'horloge et à l'état haut pendant une période. Le front montant de l'impulsion suivante provoque la remise à zéro des diviseurs.

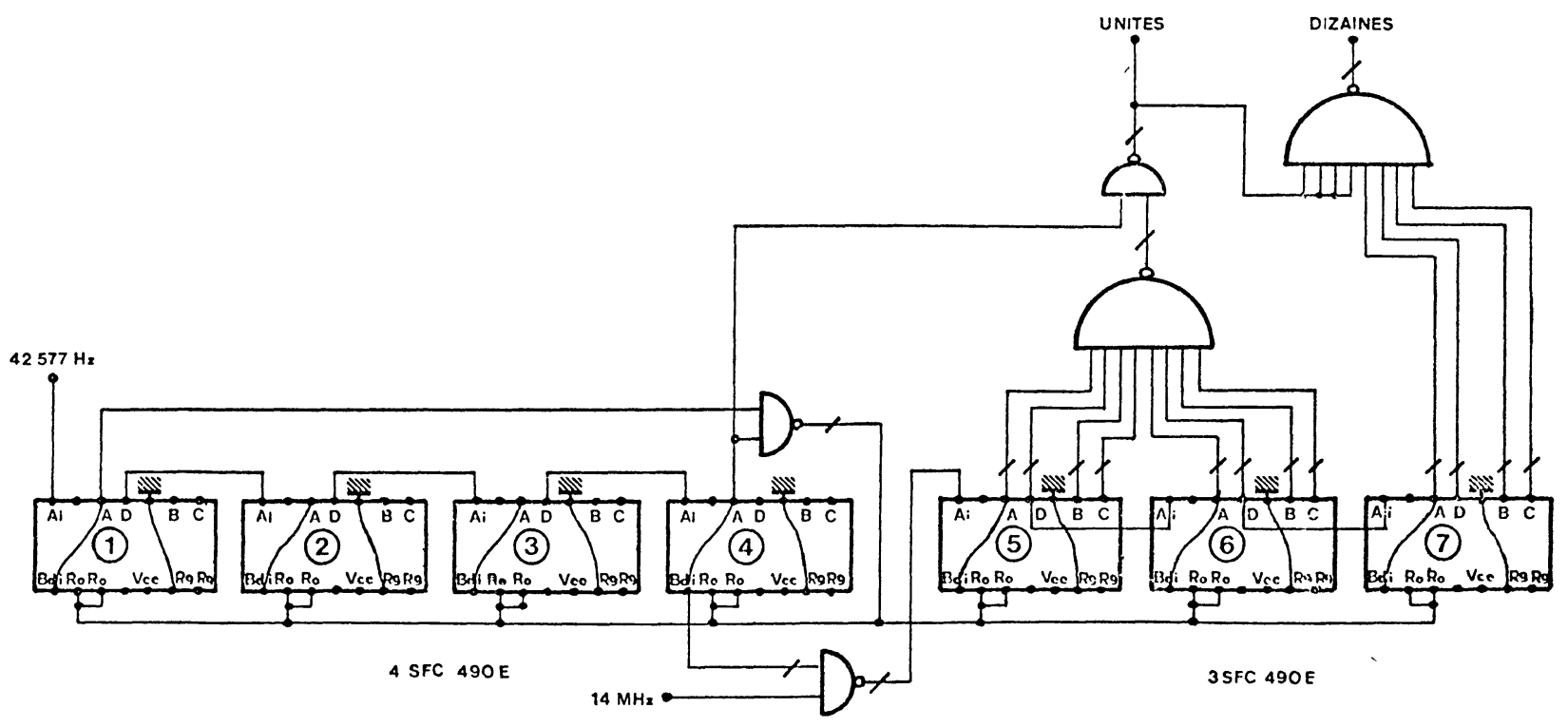

Fig. 11. - Circuits de comptage et de coïncidence. 

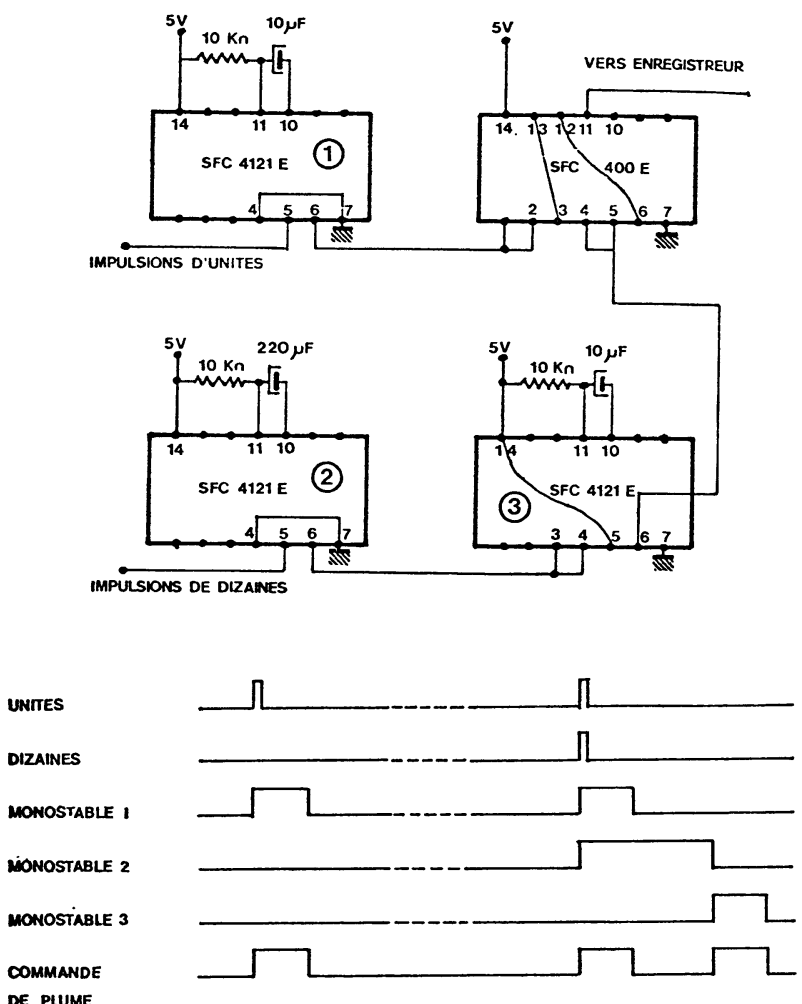

Fig. 12. - Commande du marquage.

Des circuits de coïncidence permettent de détecter le passage de la fréquence $H$. F. par un multiple entier de $4257,7 \mathrm{~Hz}$ c'est-à-dire le passage de la valeur du champ magnétique par un nombre entier de gauss ; ils permettent aussi de détecter le passage de la fréquence par un multiple entier de $42,577 \mathrm{~Hz}$ c'està-dire le passage du champ par un nombre entier de dizaines de gauss. Les impulsions correspondant aux unités comme aux dizaines de gauss sont de courte durée $(1 / 42577 \mathrm{~S})$ et indiscernables par leur amplitude ou leur largeur ; aussi commandent-elles la plume auxiliaire de l'enregistreur (HEWLETT PACKARD $7044 \mathrm{~A}$ ) par un circuit à 3 monostables (Fig. 12). Le passage par les unités se traduit par une impulsion de $0,07 \mathrm{~s}$ et le passage par les dizaines par 2 impulsions de $0,07 \mathrm{~s}$ séparées de $1,5 \mathrm{~s}$.

7. Utilisation et performances. - Les réglages de phase et de gain ayant été effectués, la tension d'affichage $V_{0}$ de la diode à capacité variable est ajustẹe pour amener le système en boucle ouverte au centre de la raie de résonance, le champ $B_{0}$ étant fixe.

Il suffit alors de boucler le système, après remise à zéro du correcteur P. I., puis de mettre en marche le marqueur, pour que l'ensemble du dispositif soit opérationnel.

Les figures 13 et 14 donnent deux exemples de spectres pour des vitesses de balayage différentes. Le marquage du spectre du radical 1-4 benzosemiquinone dissous dans l'éthanol donne pour valeur de la constante de couplage $a_{\mathrm{H}}=2,38$ gauss alors que la valeur la plus couramment donnée est de 2,368 gauss. Celui du radical 2-2-6-6 tétraméthyl pipéridone 1 oxyl dissous dans le trifluoréthanol (T. A. N. O.) conduit à une valeur de 15,7 gauss pour une valeur de 15,5 gauss obtenue par une méthode classique.

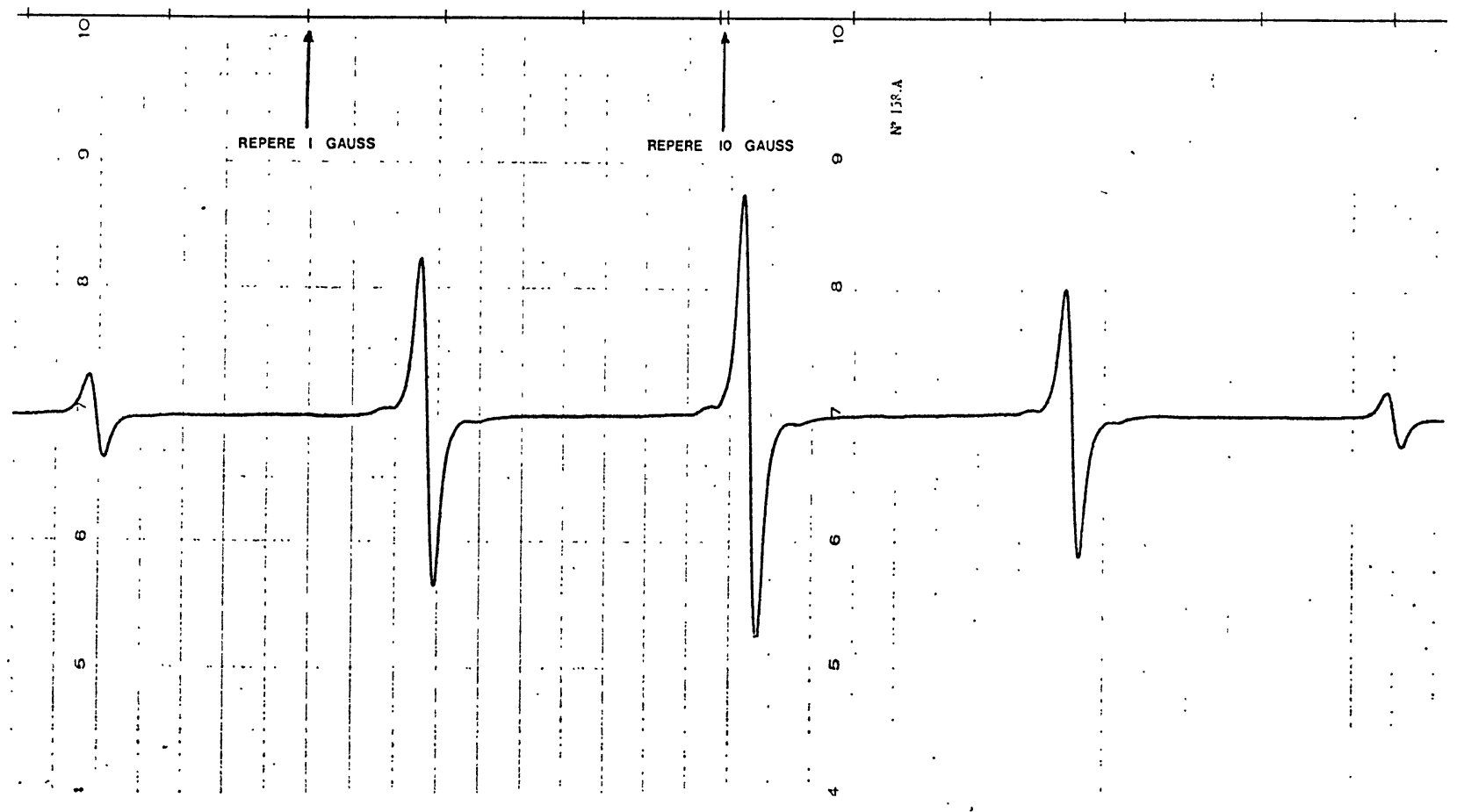

Fig. 13. - Spectre du radical 1-4 benzosemiquinone dissous dans l'éthanol. 


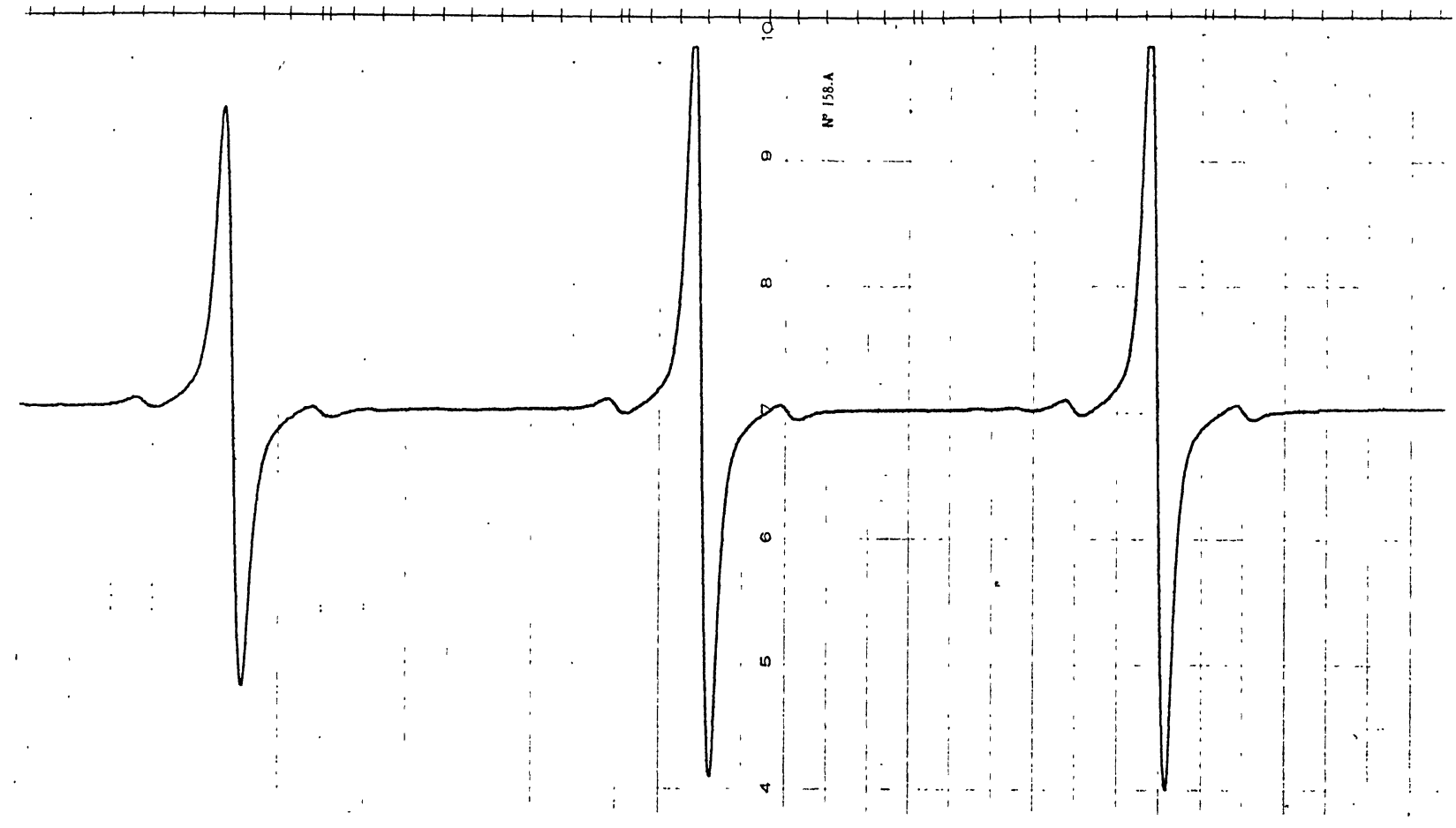

FIG. 14. - Spectre du tétraméthyl pipéridone 1 oxyl dissous dans le trifluoréthanol (TANO).

\section{Bibliographie}

[1] Hukuda, K., Iwamoto, H., Miduno, Z., Mem. Fac. Sci. Kyushu Univ. 2 (1956) 87-93.

[2] Hukuda, K., Nakahara, M., Mem. Fac. Sci. Kyushu Univ. $4(1968) 1-5$.

[3] Robinson, F. N. H., J. Sci. Instrum. 36 (1959) 481-7.

[4] Edmonds, D. T., Robinson, F. N. H., J. Sci. Instrum. 44 (1967) 475-6.

[5] Rollin, B. V., Nature, Land. 158 (1946) 669-70.

[6] Pound, R. V., KNIGHT, W. D., Rev. Sci. Instrum. 21 (1950) 219-25.
[7] Colligiani, A., Rev. Sci. Instrum. 38 (1967) 1331.

[8] Colligiani, A., Ambrosetti, R., de Lisi, P., J. Magn. Res. 20 (1975) 341-344.

[9] De CARford, P., Foulard, C., Asservissements linéaires continus (Dunod).

[10] Prudhomme, R., Automatique (Masson et Cie) tome 1, 1969.

[11] Gille, J. C., De Caulne, P., Pelegrin, M., Théorie et calcul des asservissements (Editions Dunod, Paris). 